\title{
SUPERCONVERGENCE IN VELOCITY AND PRESSURE FOR THE $3 D$ TIME-DEPENDENT NAVIER-STOKES EQUATIONS
}

\author{
FRANCISCO GUILLÉN-GONZÁLEZ, GIORDANO TIERRA
}

Departamento de Ecuaciones Diferenciales y Análisis Numérico, Universidad de Sevilla, Spain.

guillendus.es, gtierradus.es

\begin{abstract}
This work is devoted to the superconvergence in space approximation of a fully discrete scheme for the incompressible time-dependent Navier-Stokes Equations in three-dimensional domains. We discrete by Inf-Sup-stable Finite Element in space and by a semi-implicit backward Euler (linear) scheme in time.

Using an extension of the duality argument in negative-norm for elliptic linear problems (see for instance [1]) to the mixed velocity-pressure formulation of the Stokes problem, we prove some superconvergence in space results for the velocity with respect to the energy-norm, and for a weaker norm of $L^{2}\left(0, T ; L^{2}(\Omega)\right)$ type (this latter holds only for the case of Taylor-Hood approximation). On the other hand, we also obtain optimal error estimates for the pressure without imposing constraints on the time and spatial discrete parameters, arriving at superconvergence in the $H^{1}(\Omega)$-norm again for Taylor-Hood approximations. These results are numerically verified by several computational experiments, where two splitting in time schemes are also considered.
\end{abstract}

Key words: Incompressible fluids, finite elements, error estimates, uniform inf-sup Brezzi-Babuska condition, quasi-uniform meshes

AMS subject classifications: $\quad 35 Q 30,65 M 15,65 M 60,76 D 05$.

\section{Introduction}

Numerous works have been developed to study the behavior of incompressible fluids through the Navier-Stokes equations (see for instance [14], [11]). In particular, the Finite Element Method ([1], [2], [3], [4]) is a very common way of spatial approximation for the Navier-Stokes equations ([7], [9], [8], [10]).

In recent years much attention has been devoted to the formulation of efficient schemes for the Navier-Stokes equations. In [9] and [8], some time-discrete schemes are summarized showing their convergence and stability conditions.

Received: April 2, 2011. Accepted: November 12, 2011.

This work has been partially supported by the MICINN project MTM2009-12927, Spain. 
This paper focuses on a linear Euler Semi-Implicit time scheme (where the convective term is linearized) with stable finite elements in space for solving the Navier-Stokes equations for incompressible fluids (1) filling a $3 D$ domain $\Omega$ during a time interval $(0, T)$. We recall the stability of this scheme and we obtain some superconvergence in space results for the velocity $u$ and the pressure $p$ (solution of the Navier-Stokes problem (1)).

Let $\left(V_{h}, W_{h}\right)$ be conformed finite element spaces in $\left(H_{0}^{1}(\Omega)^{3}, L_{0}^{2}(\Omega)\right)$ corresponding to a regular and quasi-uniform triangulation $\mathcal{T}_{h}$ of the domain $\Omega$ with polyhedric boundary $\partial \Omega$. We assume that $\left(V_{h}, W_{h}\right)$ satisfies the following properties:

- $O\left(h^{m}\right)$-approximation in the energy norm $H^{1} \times L^{2}$.

- $\left(V_{h}, W_{h}\right)$ satisfies the so-called Babuska-Brezzi condition (BB), or discrete InfSup condition: there exists $\bar{\beta}>0$ such that

$$
\inf _{q_{h} \in W_{h}} \sup _{v_{h} \in V_{h}} \frac{\left(q_{h}, \nabla \cdot v_{h}\right)}{\left\|v_{h}\right\|_{H^{1}}\left\|q_{h}\right\|_{L^{2}}} \geq \bar{\beta} .
$$

For instance, the following choices of $\left(V_{h}, W_{h}\right)$ can be considered:

- for $m \geq 2$, the Taylor-Hood $\mathbb{P}_{m} \times \mathbb{P}_{m-1}$ finite element approximation [7, 13],

- for $m=1$, the (mini-element) $\mathbb{P}_{1}$-bubble $/ \mathbb{P}_{1}[7]$ or $\mathbb{P}_{1}$-iso $\mathbb{P}_{2} / \mathbb{P}_{1}[7]$ or $\mathbb{P}_{2} / \mathbb{P}_{0}$ [5].

More concretely, fixed a regular time partition $\left(t_{n}=n k\right)_{n=0}^{N}$ of $[0, T]$ with time step $k=T / N$, by denoting $e_{h}^{n}$ the discrete error for the velocity at time step $n$ respectively (using the discrete Stokes Projector defined in (8) as interpolator operator), we will deduce some superconvergence results in space.

Theorem 1 Assuming the $H^{2} \times H^{1}$-regularity of the Stokes Problem and under the following regularity assumptions for the exact solution $(u, p)$ of $(1)$ in $(0, T)$,

$(R 1)$

$(u, p) \in L^{\infty}\left(H^{m+1} \times H^{m}\right), \quad\left(u_{t}, p_{t}\right) \in L^{2}\left(H^{m+1} \times H^{m}\right) \quad$ and $\quad u_{t t} \in L^{2} H^{-1}$,

we obtain the error estimates for the velocity:

$$
\left\|\left(e_{h}^{n}\right)\right\|_{l^{\infty} L^{2} \cap l^{2} H^{1}} \leq C\left(k+h^{m+1}\right) .
$$

Given $X$ a Banach space, the norms

$$
\left\|\left(e^{n}\right)\right\|_{l^{\infty}(X)}=\max _{n=1, \ldots, N}\left\|e^{n}\right\|_{X}^{2} \quad \text { and } \quad\left\|\left(e^{n}\right)\right\|_{l^{2}(X)}^{2}=k \sum_{n=1}^{N}\left\|e^{n}\right\|_{X}^{2}
$$

represent the discrete version of the $L^{\infty}(0, T ; X)$ and $L^{2}(0, T ; X)$ norms for the constant by time subintervals function associated to the value $e^{n}$ at $\left(t_{n-1}, t_{n}\right)$. For brevity, we denote $L^{\infty} H^{m}$ instead of $L^{\infty}\left(0, T ; H^{m}(\Omega)\right)$ and $l^{\infty} L^{2}$ instead of $l^{\infty}\left(L^{2}(\Omega)\right)$ and so on.

On the other hand, by denoting $e_{p, h}^{n}$ as the discrete error for the pressure, we will obtain the following optimal error estimates for the pressure (without imposing constraints on the discrete time and spatial parameters $k$ and $h$ ). 
Theorem 2 Under the assumptions of Theorem 1 and assuming the additional regularity $u_{t t} \in L^{2} L^{2}$, we obtain the error estimates for the velocity and the pressure:

$$
\left\|\left(e_{h}^{n}\right)\right\|_{l^{\infty} H^{1} \cap l^{2} W^{1,6}}+\left\|\left(e_{p, h}^{n}\right)\right\|_{l^{2} H^{1}} \leq C\left(k+h^{m}\right)
$$

Note that using $\mathbb{P}_{m} / \mathbb{P}_{m-1}$ (with $m \geq 2$ ) approximation, Theorem 2 also gives superconvergence in space for the pressure. When the pressure discrete space is not included in $H^{1}(\Omega)$ (using a discontinuous approximation of the pressure) the $l^{2} H^{1}$ norm for the pressure must be changed by $l^{2} L^{6}$.

Finally, we present another superconvergence result for the velocity, only valid for $O\left(h^{m}\right)$-approximations with $m \geq 2$.

Theorem 3 Under the assumptions of Theorem 2 for $m \geq 2$ and assuming the $H^{3} \times H^{2}$-regularity of the Stokes Problem, we obtain the error estimates for the velocity:

$$
\left\|\left(e_{h}^{n}\right)\right\|_{l^{2} L^{2}} \leq C\left(k+h^{m+2}\right)
$$

whenever $k$ is small enough $\left(k \leq k_{0}\right)$.

Now, we comment two previous papers where different superconvergence results are provided.

Wheeler in [16] derived optimal error estimates for some second order semilinear parabolic partial differential equation with a coercive bilinear form. In particular, some superconvergence estimates are obtained in the $L^{2}(\Omega)$ sense. Now, we are dealing with a mixed formulation.

On the other hand, in [15], the authors developped a postprocessing technique to obtain superconvergence results for the Stokes problem. The key point is to project the finite element solution $\left(u_{h}, p_{h}\right) \in\left(V_{h}, W_{h}\right)$ related to the mesh mesh $\mathcal{T}_{h}$ to another stable finite element space $\left(V_{\tau}, W_{\tau}\right)$ with a different mesh $\mathcal{T}_{\tau}$, consisting of piecewise polynomials of higher degree $\mathbb{P}_{r}$ y $\mathbb{P}_{l}$ respectively (assume $h<<\tau$ and the relation $\tau=h^{\alpha}$ with $\alpha \in(0,1)$ between $\tau$ and $\left.h\right)$. Let $Q_{\tau}$ and $R_{\tau}$ be the $L^{2}$ projectors from $L^{2}(\Omega)$ onto $V_{\tau}$ and $W_{\tau}$, respectively. Then, in [15] the errors $u-Q_{\tau} u_{h}$ and $p-R_{\tau} p_{h}$ are analyzed, obtaining the following estimates (taking the limits $r \rightarrow \infty$ and $l \rightarrow \infty$ )

- $\left\|u-Q_{\tau} u_{h}\right\|_{L^{2}}+\left\|p-R_{\tau} p_{h}\right\|_{L^{2}} \sim O\left(h^{2}\right) \quad$ for $\mathbb{P}_{1} b \times \mathbb{P}_{1}$ - approximation.

- $\left\|u-Q_{\tau} u_{h}\right\|_{L^{2}}+\left\|p-R_{\tau} p_{h}\right\|_{L^{2}} \sim O\left(h^{4}\right) \quad$ for $\mathbb{P}_{2} \times \mathbb{P}_{1}$-approximation.

This paper is organized as follows. In Section 2 an abstract functional setting of the Navier-Stokes problem is given jointly to the existence of weak solutions. Section 3 describes the Euler Semi-Implicit scheme and their stability, meanwhile in Section 4 some superconvergence properties of the Stokes Projector are described. In sections 5, 6 and 7 we detail the proofs of the Theorems 1, 2 and 3, respectively. Furthermore, in Section 8 some numerical accurate orders are obtained by means of computational experiments. Finally, in Section 9 we present the final conclusions of the paper. 


\section{Navier-Stokes Model}

We consider the $3 D$ Navier-Stokes system, associated to the dynamics of viscous and incompressible fluids filling a bounded domain $\Omega \subset \mathbb{R}^{3}$ in a time interval $(0, T)$ :

$$
\left\{\begin{array}{cll}
u_{t}+(u \cdot \nabla) u-\nu \Delta u+\nabla p=f, & \nabla \cdot u=0, & \text { in } \Omega \times(0, T), \\
\left.u\right|_{\partial \Omega}=0 \quad t \in(0, T), & \left.u\right|_{t=0}=u_{0} & \text { in } \Omega .
\end{array}\right.
$$

Unknowns are $u: \Omega \times(0, T) \rightarrow \mathbb{R}^{3}$ the velocity field, and $p: \Omega \times(0, T) \rightarrow \mathbb{R}$ the pressure. Data are $f: \Omega \times(0, T) \rightarrow \mathbb{R}^{3}$ the external forces, and $u_{0}: \Omega \rightarrow \mathbb{R}^{3}$ the initial velocity. We denote by $\nabla$ the gradient operator, and $\Delta$ the Laplace operator. $\nu>0$ is the viscosity parameter. For simplicity, we fix $\nu=1$ (all estimates in this paper will be dependent on this viscosity parameter and the constants blow up when the viscosity vanishes).

Given $f \in C^{0}\left([0, T] ; H^{-1}(\Omega)^{3}\right)$, we consider the variational formulation of (1): Find $(u(t), p(t)) \in H_{0}^{1}(\Omega)^{3} \times L_{0}^{2}(\Omega)\left(=\left\{q \in L^{2}(\Omega) ; \int_{\Omega} q=0\right\}\right)$ such that $u(0)=u_{0}$, and

$$
\left(u_{t}(t), v\right)+a((u(t), p(t)),(v, q))+c(u(t), u(t), v)=\langle f(t), v\rangle
$$

hold a.e. $t \in(0, T)$ and for any $(v, q) \in H_{0}^{1}(\Omega)^{3} \times L_{0}^{2}(\Omega)$, where

$$
\begin{gathered}
a((u, p),(v, q))=(\nabla u, \nabla v)-(p, \nabla \cdot v)-(\nabla \cdot u, q) \quad \text { (symmetric form) } \\
c(u, w, v)=((u \cdot \nabla) w, v)+\frac{1}{2}(\nabla \cdot u, w \cdot v)=\frac{1}{2}[((u \cdot \nabla) w, v)+((u \cdot \nabla) v, w)] .
\end{gathered}
$$

Hereafter $(\cdot, \cdot)$ denotes the usual $L^{2}(\Omega)$-scalar product and $\langle\cdot, \cdot\rangle$ the $H^{-1} \times H_{0}^{1}$ duality product. Note that $c(\cdot, \cdot, \cdot)$ verifies the skew-symmetric property:

$$
c(u, v, v)=0 \quad \forall u, v \in H_{0}^{1}(\Omega)
$$

and the bounds

$$
c(u, w, v) \leq C\|u\|_{L^{2}}\|w\|_{L^{\infty} \cap W^{1,3}}\|v\|_{H^{1}}
$$

where the role of $u, w, v$ can be interchanged.

The following theorem [14] gives the existence of weak solutions of (1) (i.e. solutions of (2)).

Theorem 4 The following Inf-Sup stability condition holds: there exists $\beta>0$ such that

$$
\inf _{q \in L_{0}^{2}(\Omega)} \sup _{v \in H_{0}^{1}(\Omega)^{3}} \frac{(q, \nabla \cdot v)}{\|v\|_{H^{1}(\Omega)}\|q\|_{L^{2}(\Omega)}} \geq \beta .
$$

Moreover, problem (2) has a weak solution

$$
u \in L^{\infty}\left(0, T ; L^{2}(\Omega)^{3}\right) \cap L^{2}\left(0, T ; H^{1}(\Omega)^{3}\right), \quad p \in H^{-1}\left(0, T ; L^{2}(\Omega)\right)
$$

which verifies the following Energy Inequality:

$$
\|u(t)\|_{L^{2}}^{2}+\int_{0}^{t}\|\nabla u(s)\|_{L^{2}}^{2} \leq\left\|u_{0}\right\|_{L^{2}}^{2}+\int_{0}^{t}\|f(s)\|_{H^{-1}}^{2} \quad \forall t \in[0, T] .
$$




\section{Euler Semi-Implicit finite element scheme. Stability.}

We are going to consider the following Euler Semi-Implicit linear scheme in time and finite element approximation in space to approximate problem (2), where from now on, we denote $\delta_{t} a^{n+1}=\left(a^{n+1}-a^{n}\right) / k$ a time discrete derivative

We consider the following scheme:

Initialization: Let $u_{h}^{0} \in V_{h}$ an adequate approximation of $u_{0}$.

Step $n+1$ : Given $u_{h}^{n} \in V_{h}$, compute $\left(u_{h}^{n+1}, p_{h}^{n+1}\right) \in V_{h} \times W_{h}$ solving:

$$
\left(\delta_{t} u_{h}^{n+1}, v_{h}\right)+a\left(\left(u_{h}^{n+1}, p_{h}^{n+1}\right),\left(v_{h}, q_{h}\right)\right)+c\left(u_{h}^{n}, u_{h}^{n+1}, v_{h}\right)=\left\langle f\left(t_{n+1}\right), v_{h}\right\rangle,
$$

for any $\left(v_{h}, q_{h}\right) \in V_{h} \times W_{h}$.

Since problem (6) can be rewritten as a square algebraic linear system, uniqueness implies existence. Stability of scheme (6) is guaranteed by the following result ([11]), which in particular implies existence and uniqueness of (6):

Theorem 5 (Unconditionally Stability) Scheme (6) satisfies the following a priori estimates for velocity (which is a discrete version of (5)): for any $r=1, \ldots, N$,

$$
\left\|u_{h}^{r}\right\|_{L^{2}}^{2}+k \sum_{n=0}^{r-1}\left\|\nabla u_{h}^{n+1}\right\|_{L^{2}}^{2}+k \sum_{n=0}^{r-1}\left\|\sqrt{k} \delta_{t} u_{h}^{n+1}\right\|_{L^{2}}^{2} \leq\left\|u_{0}\right\|_{L^{2}}^{2}+\|f\|_{L^{2}\left(0, t_{r} ; H^{-1}\right)}^{2} .
$$

Furthermore, the following a priori estimate for pressure in a weighted norm holds:

$$
\left(\sqrt{k} p_{h}^{n+1}\right)_{n} \text { is bounded in } l^{4 / 3} L^{2} \quad\left(\text { i.e. } \quad\left(k \sum_{n=1}^{N}\left\|p_{h}^{n+1}\right\|_{L^{2}}^{4 / 3}\right)^{3 / 4} \leq \frac{C}{k^{1 / 2}}\right) .
$$

The weighted estimate for pressure derives for the discrete inf-sup condition (BB), weak estimates (7) (in particular, $\sqrt{k} \delta_{t} u_{h}^{n+1}$ is bounded in $l^{2} L^{2}$ ) and the following estimate for the convective term (valid in $3 D$ domains):

$$
c\left(u_{h}^{n}, u_{h}^{n+1}, v_{h}\right) \leq C\left\|u_{h}^{n}\right\|_{L^{3}}\left\|u_{h}^{n+1}\right\|_{H^{1}}\left\|v_{h}\right\|_{H^{1}} \quad \forall v_{h} \in V_{h}
$$

which implies that $c\left(u_{h}^{n}, u_{h}^{n+1}, \cdot\right)$ is bounded in $l^{4 / 3}\left(H^{-1}\right)$.

Hereafter, $C>0$ denotes a generic constant independent of $(k, h)$ and $n$.

\section{Properties of the Stokes proyector}

First of all, defining the total errors as

$$
\left(e^{n}, e_{p}^{n}\right)=\left(u\left(t_{n}\right)-u_{h}^{n}, p\left(t_{n}\right)-p_{h}^{n}\right),
$$

comparing the exact problem (2) at $t=t_{n+1}$ and the scheme (6), one arrives at $\left(\delta_{t} e^{n+1}, v_{h}\right)+a\left(\left(e^{n+1}, e_{p}^{n+1}\right),\left(v_{h}, q_{h}\right)\right)=\left(\mathcal{E}^{n+1}, v_{h}\right)-c\left(u_{h}^{n}, e^{n+1}, v_{h}\right)-c\left(e^{n}, u\left(t_{n+1}\right), v_{h}\right)$ 
where

$$
\left(\mathcal{E}^{n+1}, v_{h}\right)=-\left(u_{t}\left(t_{n+1}\right)-\delta_{t} u\left(t_{n+1}\right), v_{h}\right)-c\left(\int_{t_{n}}^{t_{n+1}} u_{t}(t), u\left(t_{n+1}\right), v_{h}\right)
$$

denotes the consistency (or truncation) error in time.

We consider as interpolation operator $\left(I_{h} u, J_{h} p\right):=S_{h}(u, p)=\left(S_{h}^{u}(u, p), S_{h}^{p}(u, p)\right)$ the Stokes Projector of each $(u, p) \in H_{0}^{1}(\Omega)^{3} \times L_{0}^{2}(\Omega)$ onto $V_{h} \times W_{h}$, i.e., $S_{h}(u, p) \in$ $V_{h} \times W_{h}$ such that

$$
a\left((u, p)-S_{h}(u, p),\left(v_{h}, q_{h}\right)\right)=0 \quad \forall\left(v_{h}, q_{h}\right) \in V_{h} \times W_{h}
$$

Then, we split the interpolation error from the discrete error (for the velocity and for the pressure) as

$$
\left(e^{n}, e_{p}^{n}\right)=\left(e_{i}^{n}, e_{p, i}^{n}\right)+\left(e_{h}^{n}, e_{p, h}^{n}\right)
$$

where

$$
\begin{array}{cl}
\left(e_{i}^{n}, e_{p, i}^{n}\right)=\left(u\left(t_{n}\right)-S_{h}^{u}\left(u\left(t_{n}\right), p\left(t_{n}\right)\right), p\left(t_{n}\right)-S_{h}^{p}\left(u\left(t_{n}\right), p\left(t_{n}\right)\right)\right) & \text { (interpolation error) } \\
\left(e_{h}^{n}, e_{p, h}^{n}\right)=\left(S_{h}^{u}\left(u\left(t_{n}\right), p\left(t_{n}\right)\right)-u_{h}^{n}, S_{h}^{p}\left(u\left(t_{n}\right), p\left(t_{n}\right)\right)-p_{h}^{n}\right) & \text { (discrete error). }
\end{array}
$$

With this choice of interpolator, the interpolation term $\left.a\left(\left(e_{i}^{n+1}, e_{p, i}^{n+1}\right)\right),\left(v_{h}, q_{h}\right)\right)$ vanish, remaining the following error equations:

$$
\left\{\begin{array}{r}
\left(\delta_{t} e_{h}^{n+1}, v_{h}\right)+a\left(\left(e_{h}^{n+1}, e_{p, h}^{n+1}\right),\left(v_{h}, q_{h}\right)\right)=\left(\mathcal{E}^{n+1}, v_{h}\right)-\left(\delta_{t} e_{i}^{n+1}, v_{h}\right) \\
-c\left(u_{h}^{n}, e^{n+1}, v_{h}\right)-c\left(e^{n}, u\left(t_{n+1}\right), v_{h}\right) .
\end{array}\right.
$$

The following approximation of the Stokes projector holds ([7]):

$$
\left\|(u, p)-S_{h}(u, p)\right\|_{H^{1} \times L^{2}} \leq C h^{m}\|u, p\|_{H^{m+1} \times H^{m}}
$$

Moreover, the following stability property of the Stokes projector will be used, either

$$
\left\|S_{h}(u, p)\right\|_{W^{1,6} \times H^{1}} \leq C\|u, p\|_{H^{2} \times H^{1}} .
$$

for continuous discrete pressure, or

$$
\left\|S_{h}(u, p)\right\|_{W^{1,6} \times L^{6}} \leq C\|u, p\|_{H^{2} \times H^{1}} .
$$

for discontinuous discrete pressure (for instance when the $\mathbb{P}_{2} / \mathbb{P}_{0}$ approximation is used). These stability results (11) and (12) can be obtained from (10) imposing the inverse inequality $\left\|v_{h}, q_{h}\right\|_{W^{1,6} \times H^{1}} \leq C h^{-1}\left\|v_{h}, q_{h}\right\|_{H^{1} \times L^{2}}$ (changing the $W^{1,6} \times$ $H^{1}$-norm by $W^{1,6} \times L^{6}$-norm in the discontinuous discrete pressure case), and comparing $S_{h}$ with an average interpolator of Clement or Scott-Zhang type. On the other hand, a more precise stability estimate like

$$
\left\|S_{h}(u, p)\right\|_{W^{1,6} \times L^{6}} \leq C\|u, p\|_{W^{1,6} \times L^{6}}
$$

was obtained in [6].

To prove superconvergence, we introduce an extension to mixed problems of the negative-norm estimates for elliptic problems by means of a Aubin-Nitsche duality argument (see for instance [1]): 
Theorem 6 Given $(f, g) \in H^{-1}(\Omega)^{3} \times L_{0}^{2}(\Omega)$, let $A^{-1}(f, g) \in H_{0}^{1}(\Omega)^{3} \times L_{0}^{2}(\Omega)$ such that

$$
a\left(A^{-1}(f, g),(v, q)\right)=\langle f, v\rangle+(g, q) \quad \forall(v, q) \in H_{0}^{1}(\Omega)^{3} \times L_{0}^{2}(\Omega) .
$$

Let $s \geq 1$ be an integer. Assume the assumptions:

1. $\left\|A^{-1}(f, g)\right\|_{H^{s+1} \times H^{s}} \leq C\|f, g\|_{H^{s-1} \times H^{s}} \quad$ (we denote $H^{0}=L^{2}$ )

2. $\inf _{\left(v_{h}, q_{h}\right) \in V_{h} \times W_{h}}\left\|A^{-1}(f, g)-\left(v_{h}, q_{h}\right)\right\|_{H^{1} \times L^{2}} \leq C h^{s}\left\|A^{-1}(f, g)\right\|_{H^{s+1} \times H^{s}}$

Then, for any $(u, p) \in H_{0}^{1}(\Omega)^{3} \times L_{0}^{2}(\Omega)$,

$$
\left\|(u, p)-S_{h}(u, p)\right\|_{\left(H^{s-1}\right)^{\prime} \times\left(H^{s} \cap L_{0}^{2}\right)^{\prime}} \leq C h^{s}\left\|(u, p)-S_{h}(u, p)\right\|_{H^{1} \times L^{2}}
$$

Proof. Let $(u, p) \in H_{0}^{1}(\Omega)^{3} \times L_{0}^{2}(\Omega)$ and $S_{h}(u, p)$ be the solution of the discrete Stokes problem (8). For any functions $(f, g) \in H^{s-1}(\Omega) \times\left(H^{s}(\Omega) \cap L_{0}^{2}(\Omega)\right)$, we consider $A^{-1}(f, g) \in\left(H^{s+1}(\Omega) \cap H_{0}^{1}(\Omega)\right)^{3} \times\left(H^{s}(\Omega) \cap L_{0}^{2}(\Omega)\right)$ as the solution of the following (adjoint) problem

$$
a\left((v, q), A^{-1}(f, g)\right)=(v, f)+(q, g), \quad \forall(v, q) \in H_{0}^{1}(\Omega)^{3} \times L_{0}^{2}(\Omega)
$$

Taking $(v, q)=(u, p)-S_{h}(u, p)$ and applying (8),

$$
\begin{aligned}
& \left(u-S_{h}^{u}(u, p), f\right)+\left(p-S_{h}^{p}(u, p), g\right)=a\left((u, p)-S_{h}(u, p), A^{-1}(f, g)\right) \\
& =a\left((u, p)-S_{h}(u, p), A^{-1}(f, g)-\left(v_{h}, w_{h}\right)\right) \quad \forall\left(v_{h}, w_{h}\right) \in V_{h} \times W_{h}
\end{aligned}
$$

Therefore, by using the continuity of $a(\cdot, \cdot)$ in $H^{1} \times L^{2}$,

$$
\begin{aligned}
& \left(u-S_{h}^{u}(u, p), f\right)+\left(p-S_{h}^{p}(u, p), g\right) \\
& \leq C\left\|(u, p)-S_{h}(u, p)\right\|_{H^{1} \times L^{2}}\left\|A^{-1}(f, g)-\left(v_{h}, w_{h}\right)\right\|_{H^{1} \times L^{2}} \quad \forall\left(v_{h}, w_{h}\right) \in V_{h} \times W_{h}
\end{aligned}
$$

Finally, by using hypotheses 1 and 2,

$$
\begin{aligned}
\left(u-S_{h}^{u}(u, p), f\right)+\left(p-S_{h}^{p}(u, p), g\right) & \leq C h^{s}\left\|(u, p)-S_{h}(u, p)\right\|_{H^{1} \times L^{2}}\left\|A^{-1}(f, g)\right\|_{H^{s+1} \times H^{s}} \\
& \leq C h^{s}\left\|(u, p)-S_{h}(u, p)\right\|_{H^{1} \times L^{2}}\|f, g\|_{H^{s-1} \times H^{s}}
\end{aligned}
$$

Since this inequality holds for any $(f, g) \in H^{s-1} \times\left(H^{s} \cap L_{0}^{2}\right)$, one arrives at (13).

We are going to use Theorem 6 jointly to the $O\left(h^{m}\right)$ approximation in the energy norm given in (10) in two particular cases:

1. ( $s=1)$ Assuming the $H^{2} \times H^{1}$-regularity of the Stokes Problem, the duality estimate given in Theorem 6 for $s=1$ yields to

$$
\left\|u-S_{h}^{u}(u, p)\right\|_{L^{2}} \leq C h\left\|(u, p)-S_{h}(u, p)\right\|_{H^{1} \times L^{2}} \leq C h^{m+1}\|(u, p)\|_{H^{m+1} \times H^{m}}
$$


2. ( $s=2$ and $m \geq 2$ ) Assuming $H^{3} \times H^{2}$-regularity of the Stokes Problem and at least $O\left(h^{2}\right)$ approximation in the energy norm, Theorem 6 for $s=2$ yields to $\left\|S_{h}^{u}(u, p)-u\right\|_{H^{-1}} \leq C h^{2}\left\|S_{h}(u, p)-(u, p)\right\|_{H^{1} \times L^{2}} \leq C h^{m+2}\|(u, p)\|_{H^{m+1} \times H^{m}}$

Since at least second order approximation in $H^{1} \times L^{2}$ must be considered, (15) is not valid for $\mathbb{P}_{1}$-bubble $/ \mathbb{P}_{1}$, or $\mathbb{P}_{1}$-iso $\mathbb{P}_{2} / \mathbb{P}_{1}$ or $\mathbb{P}_{2} / \mathbb{P}_{0}$ approximation.

$5 O\left(k+h^{m+1}\right)$ energy-norm estimates (Proof of Theorem 1).

Taking $\left(v_{h}, q_{h}\right)=2 k\left(e_{h}^{n+1},-e_{p, h}^{n+1}\right)$ in (9) the discrete pressure terms vanish hence the left hand side remains

$$
\left\|e_{h}^{n+1}\right\|_{L^{2}}^{2}-\left\|e_{h}^{n}\right\|_{L^{2}}^{2}+\left\|e_{h}^{n+1}-e_{h}^{n}\right\|_{L^{2}}^{2}+2 k\left\|\nabla e_{h}^{n+1}\right\|_{L^{2}}^{2}
$$

Then, bounding the terms of the right hand side in the following way, by using (4) to bound the convective terms and the properties of the Stokes projector (10), (11) (or (12)) and (14),

- $2 k\left(\mathcal{E}^{n+1}, e_{h}^{n+1}\right) \leq \frac{k}{4}\left\|\nabla e_{h}^{n+1}\right\|_{L^{2}}^{2}+C k^{2} \int_{t_{n}}^{t_{n+1}}\left(\left\|u_{t t}\right\|_{H^{-1}}^{2}+\left\|u_{t}\right\|_{L^{2}}^{2}\right)$

- $2 k\left(\delta_{t} e_{i}^{n+1}, e_{h}^{n+1}\right) \leq C h^{m+1}\left\|\int_{t_{n}}^{t_{n+1}}\left(u_{t}, p_{t}\right)\right\|_{H^{m+1} \times H^{m}}\left\|e_{h}^{n+1}\right\|_{L^{2}}$

$$
\leq \frac{k}{4}\left\|\nabla e_{h}^{n+1}\right\|_{L^{2}}^{2}+C h^{2(m+1)} \int_{t_{n}}^{t_{n+1}}\left\|u_{t}, p_{t}\right\|_{H^{m+1} \times H^{m}}^{2}
$$

- $2 k c\left(e^{n}, u\left(t_{n+1}\right), e_{h}^{n+1}\right) \leq C k\left\|e_{i}^{n}+e_{h}^{n}\right\|_{L^{2}}\left\|u\left(t_{n+1}\right)\right\|_{L^{\infty} \cap W^{1,3}}\left\|e_{h}^{n+1}\right\|_{H^{1}}$ $\leq \frac{k}{4}\left\|\nabla e_{h}^{n+1}\right\|_{L^{2}}^{2}+C k h^{2(m+1)}\left\|u\left(t_{n}\right), p\left(t_{n}\right)\right\|_{H^{m+1} \times H^{m}}^{2}\left\|u\left(t_{n+1}\right)\right\|_{H^{2}}^{2}+$ $C k\left\|u\left(t_{n+1}\right)\right\|_{H^{2}}^{2}\left\|e_{h}^{n}\right\|_{L^{2}}^{2}$

- $c\left(u_{h}^{n}, e_{h}^{n+1}, e_{h}^{n+1}\right)=0$ owing to (3),

- $2 k c\left(u_{h}^{n}, e_{i}^{n+1}, e_{h}^{n+1}\right) \leq 2 k c\left(S_{h}^{u}\left(u\left(t_{n}\right), p\left(t_{n}\right)\right), e_{i}^{n+1}, e_{h}^{n+1}\right)+2 k c\left(e_{h}^{n}, e_{i}^{n+1}, e_{h}^{n+1}\right)$

$\leq C k\left\|S_{h}^{u}\left(u\left(t_{n}\right), p\left(t_{n}\right)\right)\right\|_{L^{\infty} \cap W^{1,3}}\left\|e_{i}^{n+1}\right\|_{L^{2}}\left\|e_{h}^{n+1}\right\|_{H^{1}}+C k\left\|e_{i}^{n+1}\right\|_{L^{\infty} \cap W^{1,3}}\left\|e_{h}^{n}\right\|_{L^{2}}\left\|e_{h}^{n+1}\right\|_{H^{1}}$

$\leq \frac{k}{4}\left\|\nabla e_{h}^{n+1}\right\|_{L^{2}}^{2}+C k h^{2(m+1)}\left\|u\left(t_{n}\right), p\left(t_{n}\right)\right\|_{H^{2} \times H^{1}}^{2}\left\|u\left(t_{n+1}\right), p\left(t_{n+1}\right)\right\|_{H^{m+1} \times H^{m}}^{2}$ $+C k\left\|u\left(t_{n+1}\right), p\left(t_{n+1}\right)\right\|_{H^{2} \times H^{1}}^{2}\left\|e_{h}^{n}\right\|_{L^{2}}^{2}$,

one arrives at

$$
\begin{aligned}
& \left\|e_{h}^{n+1}\right\|_{L^{2}}^{2}-\left\|e_{h}^{n}\right\|_{L^{2}}^{2}+\left\|e_{h}^{n+1}-e_{h}^{n}\right\|_{L^{2}}^{2}+k\left\|\nabla e_{h}^{n+1}\right\|_{L^{2}}^{2} \leq C k^{2} \int_{t_{n}}^{t_{n+1}}\left(\left\|u_{t t}\right\|_{H^{-1}}^{2}+\left\|u_{t}\right\|_{L^{2}}^{2}\right) \\
& \quad+C h^{2(m+1)} \int_{t_{n}}^{t_{n+1}}\left\|u_{t}, p_{t}\right\|_{H^{m+1} \times H^{m}}^{2} \\
& \quad+C k h^{2(m+1)}\left\|u\left(t_{n}\right), p\left(t_{n}\right)\right\|_{H^{2} \times H^{1}}^{2}\left\|u\left(t_{n+1}\right), p\left(t_{n+1}\right)\right\|_{H^{m+1} \times H^{m}}^{2} \\
& \quad+C k\left\|u\left(t_{n+1}\right), p\left(t_{n+1}\right)\right\|_{H^{2} \times H^{1}}^{2}\left\|e_{h}^{n}\right\|_{L^{2}}^{2}
\end{aligned}
$$


Adding from $n=0$ to $r-1$, for each $r=1, \ldots, N$, and applying the discrete Gronwall's Lemma ([10]), one gets:

$$
\begin{aligned}
& \left\|e_{h}^{r}\right\|_{L^{2}}^{2}+k \sum_{n=0}^{r-1}\left\|\nabla e_{h}^{n+1}\right\|_{L^{2}}^{2} \leq e^{D}\left(\left\|e_{h}^{0}\right\|_{L^{2}}^{2}+C k^{2} \int_{0}^{t_{r}}\left(\left\|u_{t t}\right\|_{H^{-1}}^{2}+\left\|u_{t}\right\|_{L^{2}}^{2}\right)\right. \\
& \left.\quad+C h^{2(m+1)} \int_{0}^{t_{r}}\left\|u_{t}, p_{t}\right\|_{H^{m+1} \times H^{m}}^{2}+C h^{2(m+1)}\|u, p\|_{L^{\infty}\left(0, t_{r} ; H^{m+1} \times H^{m}\right)}^{2}\right)
\end{aligned}
$$

where $D>0$ depends on $\|u, p\|_{L^{\infty}\left(0, t_{r} ; H^{2} \times H^{1}\right)}^{2}$. Therefore, under the following regularity assumptions:

$(u, p) \in L^{\infty}\left(H^{m+1} \times H^{m}\right), \quad\left(u_{t}, p_{t}\right) \in L^{2}\left(H^{m+1} \times H^{m}\right) \quad$ and $\quad u_{t t} \in L^{2} H^{-1}$,

we obtain the error estimates in velocity:

$$
\left\|\left(e_{h}^{n}\right)\right\|_{l^{\infty} L^{2} \cap l^{2} H^{1}} \leq C\left(k+h^{m+1}\right) .
$$

$y$ the interpolator properties, we also obtain the following optimal error estimate in $l^{\infty} L^{2}$ for the total error:

$$
\left\|\left(u\left(t_{n}\right)-u_{h}^{n}\right)\right\|_{l^{\infty} L^{2}} \leq C\left(k+h^{m+1}\right),
$$

but estimate (17) respect to the $H^{1}(\Omega)$-norm can not be conserved, because the interpolation error has only order $O\left(h^{m}\right)$ in the $H^{1}$-norm. Therefore, we have obtained superconvergence for the discrete velocity approximation in the $l^{2} H^{1}$-norm.

\section{Error Estimates for the Pressure (Proof of Theorem 2).}

Considering the Stokes Projector $S_{h}(u, p)$ and assuming regularity hypothesis (16), we have already obtained the superconvergence error estimates in energy-norms:

$$
\left\|\left(e_{h}^{n}\right)\right\|_{l^{\infty} L^{2} \cap l^{2} H^{1}} \leq C\left(k+h^{m+1}\right)
$$

which is valid either for $m=1$ and $\mathbb{P}_{1}$-bubble $/ \mathbb{P}_{1}$ or $\mathbb{P}_{1}$-iso $\mathbb{P}_{2} / \mathbb{P}_{1}$ or $\mathbb{P}_{2} / \mathbb{P}_{0}$ approximation or for $m \geq 2$ and $\mathbb{P}_{m} / \mathbb{P}_{m-1}$ approximation. But, these energy-norm estimates are not sufficient to get optimal error estimates for the pressure.

Now, the main idea is to get error estimates for more regular norms. Introducing the notation $c(u, v, w)=(C(u, v), w)$, the error equations (9) can be written as follows:

$$
\left(\delta_{t} e_{h}^{n+1}, v_{h}\right)+a\left(\left(e_{h}^{n+1}, e_{p, h}^{n+1}\right),\left(v_{h}, q_{h}\right)\right)=\left(g, v_{h}\right)
$$

where $g=\mathcal{E}^{n+1}+\delta_{t} e_{i}^{n+1}+C\left(u_{h}^{n}, e^{n+1}\right)+C\left(e^{n}, u\left(t_{n+1}\right)\right)$. In particular, from the stability property (26),

$$
\left\|e_{h}^{n+1}, e_{p, h}^{n+1}\right\|_{W^{1,6} \times H^{1}}^{2} \leq C\|g\|_{L^{2}}^{2}+C\left\|\delta_{t} e_{h}^{n+1}\right\|_{L^{2}}^{2} .
$$


On the other hand, taking $v_{h}=2 \delta_{t} e_{h}^{n+1}\left(\right.$ and $\left.q_{h}=0\right)$ in (18)

$$
\left\|\delta_{t} e_{h}^{n+1}\right\|_{L^{2}}^{2}+\delta_{t}\left\|e_{h}^{n+1}\right\|_{H^{1}}^{2}+\frac{1}{k}\left\|e_{h}^{n+1}-e_{h}^{n}\right\|_{H^{1}}^{2} \leq C\|g\|_{L^{2}}^{2}
$$

Combining the two previous inequalities as $\alpha(19)+(20)$, with $\alpha$ small enough in order to absorb the term $C \alpha\left\|\delta_{t} e_{h}^{n+1}\right\|_{L^{2}}^{2}$ at the right hand-side, one has

$$
\begin{aligned}
& \frac{1}{2}\left\|\delta_{t} e_{h}^{n+1}\right\|_{L^{2}}^{2}+\delta_{t}\left\|e_{h}^{n+1}\right\|_{H^{1}}^{2}+\alpha\left\|e_{h}^{n+1}, e_{p, h}^{n+1}\right\|_{W^{1,6} \times H^{1}}^{2}+\frac{1}{k}\left\|e_{h}^{n+1}-e_{h}^{n}\right\|_{H^{1}}^{2} \\
& \leq C\|g\|_{L^{2}}^{2} \leq C\left(\left\|\mathcal{E}^{n+1}\right\|_{L^{2}}^{2}+\left\|\delta_{t} e_{i}^{n+1}\right\|_{L^{2}}^{2}+\left\|C\left(e^{n}, u\left(t_{n+1}\right)\right)\right\|_{L^{2}}^{2}+\left\|C\left(u_{h}^{n}, e^{n+1}\right)\right\|_{L^{2}}^{2}\right) .
\end{aligned}
$$

We bound the nonlinear terms as follows:

$$
\begin{aligned}
& \left\|C\left(e^{n}, u\left(t_{n+1}\right)\right)\right\|_{L^{2}}^{2} \leq\left\|e^{n}\right\|_{H^{1}}^{2}\left\|u\left(t_{n+1}\right)\right\|_{W^{1,3} \cap L^{\infty}}^{2} \\
& \leq C\left\|e_{h}^{n}\right\|_{H^{1}}^{2}+C h^{2 m}\left\|u\left(t_{n}\right), p\left(t_{n}\right)\right\|_{H^{m+1} \times H^{m}}^{2} \leq C\left\|e_{h}^{n}\right\|_{H^{1}}^{2}+C h^{2 m}
\end{aligned}
$$

and

$$
C\left(u_{h}^{n}, e^{n+1}\right)=C\left(S_{h}^{u} u\left(t_{n}\right), e^{n+1}\right)+C\left(e_{h}^{n}, e_{i}^{n+1}\right)+C\left(e_{h}^{n}, e_{h}^{n+1}\right)
$$

bounding each term as:

$$
\begin{gathered}
\left\|C\left(S_{h}^{u} u\left(t_{n}\right), e^{n+1}\right)\right\|_{L^{2}}^{2} \leq C\left\|e_{h}^{n+1}\right\|_{H^{1}}^{2}+C h^{2 m}\left\|u\left(t_{n+1}\right), p\left(t_{n+1}\right)\right\|_{H^{m+1} \times H^{m}}^{2} \\
\quad \leq C\left(\left\|e_{h}^{n+1}-e_{h}^{n}\right\|_{H^{1}}^{2}+\left\|e_{h}^{n}\right\|_{H^{1}}^{2}\right)+C h^{2 m} \\
\begin{aligned}
\left\|C\left(e_{h}^{n}, e_{i}^{n+1}\right)\right\|_{L^{2}}^{2} & \leq C\left\|e_{h}^{n}\right\|_{H^{1}}^{2}\left\|e_{i}^{n+1}\right\|_{W^{1,3} \cap L^{\infty}}^{2} \leq C\left\|e_{h}^{n}\right\|_{H^{1}}^{2}\left\|u\left(t_{n+1}\right), p\left(t_{n+1}\right)\right\|_{H^{2} \times H^{1}}^{2} \\
& \leq C\left\|e_{h}^{n}\right\|_{H^{1}}^{2}
\end{aligned} \\
\left\|C\left(e_{h}^{n}, e_{h}^{n+1}\right)\right\|_{L^{2}}^{2} \leq C\left\|\nabla e_{h}^{n} \cdot e_{h}^{n+1}\right\|_{L^{2}}^{2}+C\left\|\nabla e_{h}^{n+1} \cdot e_{h}^{n}\right\|_{L^{2}}^{2}
\end{gathered}
$$

where

$$
\begin{aligned}
& \left\|\nabla e_{h}^{n} \cdot e_{h}^{n+1}\right\|_{L^{2}}^{2} \leq\left\|\nabla e_{h}^{n}\right\|_{L^{3}}^{2}\left\|e_{h}^{n+1}\right\|_{L^{6}}^{2} \leq\left\|\nabla e_{h}^{n}\right\|_{L^{6}}\left\|\nabla e_{h}^{n}\right\|_{L^{2}}\left\|e_{h}^{n+1}\right\|_{H^{1}}^{2} \\
& \leq \varepsilon\left\|e_{h}^{n}\right\|_{W^{1,6}}^{2}+C_{\varepsilon}\left\|e_{h}^{n}\right\|_{H^{1}}^{2}\left\|e_{h}^{n+1}\right\|_{H^{1}}^{4} \leq \varepsilon\left\|e_{h}^{n}\right\|_{W^{1,6}}^{2}+C_{\varepsilon}\left\|e_{h}^{n}\right\|_{H^{1}}^{2}
\end{aligned}
$$

and for the same manner

$$
\begin{aligned}
& \left\|e_{h}^{n} \cdot \nabla e_{h}^{n+1}\right\|_{L^{2}}^{2} \leq \varepsilon\left\|e_{h}^{n+1}\right\|_{W^{1,6}}^{2}+C_{\varepsilon}\left\|e_{h}^{n+1}\right\|_{H^{1}}^{2}\left\|e_{h}^{n}\right\|_{H^{1}}^{4} \\
& \leq \varepsilon\left\|e_{h}^{n+1}\right\|_{W^{1,6}}^{2}+C_{\varepsilon}\left(\left\|e_{h}^{n+1}-e_{h^{n}}^{n}\right\|_{H^{1}}^{2}+\left\|e_{h}^{n}\right\|_{H^{1}}^{2}\right)
\end{aligned}
$$

where $C_{\varepsilon}=O(1 / \varepsilon)$. On the other hand,

$$
\left\|C\left(e_{h}^{n}, e_{h}^{n+1}\right)\right\|_{L^{2}}^{2} \leq \varepsilon\left(\left\|e_{h}^{n+1}\right\|_{W^{1,6}}^{2}+\left\|e_{h}^{n}\right\|_{W^{1,6}}^{2}\right)+C_{\varepsilon} K(n)\left\|e_{h}^{n}\right\|_{H^{1}}^{2}
$$

where

$$
K(n)=\left\|e_{h}^{n+1}\right\|_{H^{1}}^{4}+\left\|e_{h}^{n}\right\|_{H^{1}}^{2}\left\|e_{h}^{n+1}\right\|_{H^{1}}^{2} .
$$

Therefore, plugging all these estimates in (21) one has

$$
\begin{aligned}
& \frac{1}{2}\left\|\delta_{t} e_{h}^{n+1}\right\|_{L^{2}}^{2}+\delta_{t}\left\|e_{h}^{n+1}\right\|_{H^{1}}^{2}+\alpha\left\|e_{h}^{n+1}, e_{p, h}^{n+1}\right\|_{W^{1,6} \times H^{1}}^{2}+\frac{1}{k}\left\|e_{h}^{n+1}-e_{h}^{n}\right\|_{H^{1}}^{2} \\
& \leq C k \int_{t_{n}}^{t_{n+1}}\left(\left\|u_{t t}\right\|_{L^{2}}^{2}+\left\|u_{t}\right\|_{H^{1}}^{2}\right)+C \frac{h^{2(m+1)}}{k} \int_{t_{n}}^{t_{n+1}}\left\|u_{t}, p_{t}\right\|_{H^{m+1} \times H^{m}}^{2} \\
& +C_{\varepsilon}\left(\left\|e_{h}^{n}\right\|_{H^{1}}^{2}+\left\|e_{h}^{n+1}-e_{h}^{n}\right\|_{H^{1}}^{2}\right)+C h^{2 m}+\varepsilon\left(\left\|e_{h}^{n+1}\right\|_{W^{1,6}}^{2}+\left\|e_{h}^{n}\right\|_{W^{1,6}}^{2}\right)+C_{\varepsilon} K(n)\left\|e_{h}^{n}\right\|_{H^{1}}^{2}
\end{aligned}
$$


We take first $\varepsilon$ small enough with respect to $\alpha$ and second impose small enough time steps $k$ with respect to $C_{\varepsilon}$ (that is $k \leq k_{0}$ ), in order to absorb some RHS terms. Then, multiplying by $k$, adding from $n=0, \cdots, r(r<N)$, assuming the additional regularity $u_{t t} \in L^{2}\left(L^{2}\right)$ and using the discrete Gronwall's Lemma, one gets:

$$
\begin{aligned}
& \left\|\left(\delta_{t} e_{h}^{n+1}\right)\right\|_{l^{2} L^{2}}^{2}+\left\|\left(e_{h}^{n+1}\right)\right\|_{l^{\infty} H^{1}}^{2}+\left\|\left(e_{h}^{n+1}, e_{p, h}^{n+1}\right)\right\|_{l^{2}\left(W^{1,6} \times H^{1}\right)}^{2} \\
& \leq C \mathrm{e}^{k \sum_{n} K(n)}\left(\left\|e_{h}^{0}\right\|_{H^{1}}^{2}+\varepsilon\left\|e_{h}^{0}\right\|_{W^{1,6}}^{2}+k^{2}+h^{2 m}\right) .
\end{aligned}
$$

At this point, we split the argument into two cases:

1. Estimates assuming $h<<f(k)$ ( $h$ small enough with respect to $k$ ):

By using $k \sum_{n}\left\|e_{h}^{n}\right\|_{H^{1}}^{2} \leq C\left(k^{2}+h^{2(m+1)}\right)$ given in Theorem 1 (and in particular $\left.\left\|e_{h}^{n}\right\|_{H^{1}}^{2} \leq C\left(k+h^{2(m+1)} / k\right)\right)$, one has

$$
k \sum_{n} K(n) \leq C\left(k+\frac{h^{2(m+1)}}{k}\right)\left(k^{2}+h^{2(m+1)}\right),
$$

hence one can bound

$$
k \sum_{n} K(n) \leq C
$$

under the hypothesis

$$
\frac{h^{4(m+1)}}{k} \leq C
$$

2. Estimates assuming $k<<g(h)$ ( $k$ small enough with respect to $h$ ):

By using $\left\|e_{h}^{n}\right\|_{L^{2}}^{2} \leq C\left(k^{2}+h^{2(m+1)}\right)$ for each $n$ (as consequence of Theorem $1)$, and the inverse inequality $\left\|e_{h}\right\|_{H^{1}} \leq C h^{-1}\left\|e_{h}\right\|_{L^{2}}$, one has

$$
k \sum_{n} K(n) \leq C \frac{1}{h^{2}}\left(k^{2}+h^{2(m+1)}\right)^{2},
$$

hence one can bound

$$
k \sum_{n} K(n) \leq C
$$

under the hypothesis

$$
\frac{k^{4}}{h^{2}} \leq C
$$

Then, combining both arguments, we deduce the following estimates:

$$
\left\|\left(\delta_{t} e_{h}^{n+1}\right)\right\|_{l^{2} L^{2}}^{2}+\left\|\left(e_{h}^{n+1}\right)\right\|_{l^{\infty} H^{1} \cap l^{2} W^{1,6}}^{2}+\left\|\left(e_{p, h}^{n+1}\right)\right\|_{l^{2} H^{1}}^{2} \leq C\left(k^{2}+h^{2 m}\right)
$$

without imposing constraints on the discrete parameters $(h, k)$, because for any choice of $(h, k)$, either $(H 1)$ or $(H 2)$ holds. 
ote that estimate (22), for $\mathbb{P}_{m} / \mathbb{P}_{m-1}(m \geq 2)$ approximation, also gives superconvergence in space for the pressure in $l^{2} H^{1}$ and optimal estimate for the velocity in $l^{\infty} H^{1}$. On the other hand, when $m=1$ and $\mathbb{P}_{1}$-bubble/ $\mathbb{P}_{1}$ or $\mathbb{P}_{1}$-iso $\mathbb{P}_{2} / \mathbb{P}_{1}$ is used, we have optimal estimate for the velocity in $l^{\infty} H^{1}$ and for the pressure in $l^{2} H^{1}$ (changing this last norm by $l^{2} L^{6}$ when $\mathbb{P}_{2} / \mathbb{P}_{0}$ is used)

$t$ is possible to obtain estimate (22) without using the superconvergence results in space for the velocity given in Theorem 1 (even for $m=1$ ), changing the constraint $(H 1)$ by the following one

$$
h^{4 m} / k \leq C
$$

Indeed for $m=1$ (and, for instance, $\mathbb{P}_{1} b / \mathbb{P}_{1}$ or $\mathbb{P}_{2} / \mathbb{P}_{0}$ approximation), $(H 1)^{\prime}$ : $h^{4} / k \leq C$ and $(H 2): k^{4} / h^{2} \leq C$ are both verified by the choice $h=k^{\delta}$ with $\delta \in[1 / 4,2]$, therefore at least one constraint $(H 1)^{\prime}$ or $(H 2)$ holds.

\section{$7 O\left(k+h^{m+2}\right)$ in $l^{2}\left(L^{2}\right)$ for Taylor-Hood (Proof of Theorem 3).}

In this section, by using a duality argument and the improved interpolation error (15) (only valid for the Taylor-Hood approximation), the order accuracy in space respect the $l^{2} L^{2}$-norm will be improved.

The idea is to take $A_{h}^{-1} e_{h}^{n+1}:=S_{h} A^{-1} e_{h}^{n+1}$ as test function in (9), where $S_{h}$ is the Stokes projector defined in (8) and $A^{-1}$ is the continuous Stokes resolvent, i.e. $A^{-1} f=\left(A_{u}^{-1} f, A_{p}^{-1} f\right) \in H_{0}^{1}(\Omega)^{3} \times L_{0}^{2}(\Omega)$ such that

$$
a\left(A^{-1} f,(v, q)\right)=(f, v) \quad \forall(v, q) \in H_{0}^{1}(\Omega)^{3} \times L_{0}^{2}(\Omega) .
$$

Therefore, $A_{h}^{-1} e_{h}^{n+1}=\left(A_{h, u}^{-1} e_{h}^{n+1}, A_{h, p}^{-1} e_{h}^{n+1}\right) \in V_{h} \times W_{h}$ is the solution of the discrete Stokes problem:

$$
a\left(A_{h}^{-1} e_{h}^{n+1},\left(v_{h}, q_{h}\right)\right)=\left(e_{h}^{n+1}, v_{h}\right) \quad \forall\left(v_{h}, q_{h}\right) \in V_{h} \times W_{h} .
$$

In particular, since $e_{h}^{n}$ and $e_{h}^{n+1}$ verify the discrete free-divergence condition, the following equalities hold:

$$
\begin{aligned}
& a\left(A_{h}^{-1} e_{h}^{n+1},\left(e_{h}^{n+1}, e_{p, h}^{n+1}\right)\right)=\left\|e_{h}^{n+1}\right\|_{L^{2}}^{2}, \\
& \left(\delta_{t} e_{h}^{n+1}, A_{h, u}^{-1} e_{h}^{n+1}\right)=\frac{1}{2} \delta_{t}\left\|\nabla A_{h, u}^{-1} e_{h}^{n+1}\right\|_{L^{2}}^{2}+\frac{1}{2} k\left\|\nabla \delta_{t} A_{h, u}^{-1} e_{h}^{n+1}\right\|_{L^{2}}^{2}
\end{aligned}
$$

Moreover, by using the $H^{2} \times H^{1}$ regularity of this Stokes Problem and the approximation property of $S_{h}$, the following approximation property holds:

$$
\left\|A_{h}^{-1} e_{h}^{n+1}-A^{-1} e_{h}^{n+1}\right\|_{H^{1} \times L^{2}} \leq C h\left\|e_{h}^{n+1}\right\|_{L^{2}},
$$

hence the following inequality can be easily deduced:

$$
\forall g \in L^{2}(\Omega), \quad\left(g, \nabla A_{h, u}^{-1} e_{h}^{n+1}\right) \leq C\left(h\|g\|_{L^{2}}+C\|g\|_{\left(H^{1}\right)^{\prime}}\right)\left\|e_{h}^{n+1}\right\|_{L^{2}}
$$


Using the stability property of the Stokes projector (11) and the $H^{2} \times H^{1}$ regularity of the Stokes Problem, one has the stability property

$$
\left\|A_{h}^{-1} e_{h}^{n+1}\right\|_{W^{1,6} \times H^{1}} \leq C\left\|e_{h}^{n+1}\right\|_{L^{2}} .
$$

Thus, taking $\left(v_{h}, q_{h}\right)=2 k A_{h}^{-1} e_{h}^{n+1}$ in (9), using (23), (24) the left hand side remains

$$
\left\|\nabla A_{h}^{-1} e_{h}^{n+1}\right\|_{L^{2}}^{2}-\left\|\nabla A_{h}^{-1} e_{h}^{n}\right\|_{L^{2}}^{2}+\left\|\nabla A_{h}^{-1} e_{h}^{n+1}-\nabla A_{h}^{-1} e_{h}^{n}\right\|_{L^{2}}^{2}+2 k\left\|e_{h}^{n+1}\right\|_{L^{2}}^{2}
$$

and bounding the right hand side as follows (taking into account the regularity for the exact solution (16) and bounds (15), (25) and (26)):

- $2 k\left(\delta_{t} e_{i}^{n+1}, A_{h, u}^{-1} e_{h}^{n+1}\right) \leq C\left\|e_{i}\left(\int_{t_{n}}^{t_{n+1}} u_{t} d t\right)\right\|_{H^{-1}}\left\|A_{h, u}^{-1} e_{h}^{n+1}\right\|_{L^{2}}$

$$
\leq \frac{k}{3}\left\|e_{h}^{n+1}\right\|_{L^{2}}^{2}+C h^{2(m+2)} \int_{t_{n}}^{t_{n+1}}\left\|u_{t}, p_{t}\right\|_{H^{m+1} \times H^{m}}^{2}
$$

- $2 k c\left(e^{n}, u\left(t_{n+1}\right), A_{h, u}^{-1} e_{h}^{n+1}\right) \leq C k\left\|A_{h, u}^{-1} e_{h}^{n+1}\right\|_{H^{1}}\left\|e_{h}^{n}\right\|_{L^{2}}+C k\left\|e_{h}^{n+1}\right\|_{L^{2}}\left(h\left\|e_{i}^{n}\right\|_{L^{2}}+\right.$ $\left\|e_{i}^{n}\right\|_{\left.\left(H^{1}\right)^{\prime}\right)}$

$$
\leq \frac{k}{2}\left\|e_{h}^{n}\right\|_{L^{2}}^{2}+\frac{k}{3}\left\|e_{h}^{n+1}\right\|_{L^{2}}^{2}+C k\left\|\nabla A_{h, u}^{-1} e_{h}^{n+1}\right\|_{L^{2}}^{2}+C k h^{2(m+2)}\left\|u\left(t_{n}\right), p\left(t_{n}\right)\right\|_{H^{m+1} \times H^{m}}^{2}
$$

- $2 k c\left(u_{h}^{n}, e^{n+1}, A_{h, u}^{-1} e_{h}^{n+1}\right)=2 k c\left(S_{h}^{u}\left(u\left(t_{n}\right), p\left(t_{n}\right)\right), e^{n+1}, A_{h, u}^{-1} e_{h}^{n+1}\right)+2 k c\left(e_{h}^{n}, e^{n+1}, A_{h, u}^{-1} e_{h}^{n+1}\right)$

$\leq C k\left\|e_{h}^{n+1}\right\|_{L^{2}}\left(\left\|A_{h, u}^{-1} e_{h}^{n+1}\right\|_{H^{1}}+h\left\|e_{i}^{n+1}\right\|_{L^{2}}+\left\|e_{i}^{n+1}\right\|_{\left(H^{1}\right)^{\prime}}\right)+C k\left\|e_{h}^{n}\right\|_{H^{1}}\left\|e^{n+1}\right\|_{L^{2}}\left\|e_{h}^{n+1}\right\|_{L^{2}}$

$\leq \frac{k}{3}\left\|e_{h}^{n+1}\right\|_{L^{2}}^{2}+C k\left\|\nabla A_{h, u}^{-1} e_{h}^{n+1}\right\|_{L^{2}}^{2}+C k h^{2(m+2)}\left\|u\left(t_{n+1}\right), p\left(t_{n+1}\right)\right\|_{H^{m+1} \times H^{m}}^{2}$ $+C k\left(k+h^{m+1}\right)^{2}\left\|e_{h}^{n}\right\|_{H^{1}}^{2}$

Then,

$$
\begin{aligned}
& \left\|\nabla A_{h}^{-1} e_{h}^{n+1}\right\|_{L^{2}}^{2}-\left\|\nabla A_{h}^{-1} e_{h}^{n}\right\|_{L^{2}}^{2}+\left\|\nabla A_{h}^{-1} e_{h}^{n+1}-\nabla A_{h}^{-1} e_{h}^{n}\right\|_{L^{2}}^{2}+k\left\|e_{h}^{n+1}\right\|_{L^{2}}^{2} \\
& \quad \leq C k^{2} \int_{t_{n}}^{t_{n+1}}\left(\left\|u_{t t}\right\|_{H^{-1}}^{2}+\left\|u_{t}\right\|_{L^{2}}^{2}\right)+C h^{2(m+2)} \int_{t_{n}}^{t_{n+1}}\left\|u_{t}, p_{t}\right\|_{H^{m+1} \times H^{m}}^{2}+\frac{k}{2}\left\|e_{h}^{n}\right\|_{L^{2}}^{2} \\
& \quad+C k h^{2(m+2)}+C\left(k^{2}+h^{2(m+1)}\right) k\left\|e_{h}^{n}\right\|_{H^{1}}^{2}+C k\left\|\nabla A_{h, u}^{-1} e_{h}^{n+1}\right\|_{L^{2}}^{2}
\end{aligned}
$$

Combining the estimate $k \sum_{n}\left\|e_{h}^{n}\right\|_{H^{1}}^{2} \leq C\left(k^{2}+h^{2(m+1)}\right)$ given in Theorem 1 with the generalized discrete Gronwall's Lemma, one can obtain for $k$ small enough (i.e., $\left.k \leq k_{0}\right)$ :

$$
\left\|\left(e_{h}^{n}\right)\right\|_{l^{2}\left(L^{2}\right)} \leq C\left(k+h^{m+2}\right) .
$$

he total error estimate can not be improved to $O\left(h^{m+2}\right)$ in $l^{2}\left(L^{2}\right)$ (but it is possible to improve it in the $l^{2}\left(H^{-1}\right)$-norm). That means superconvergence in $l^{2}\left(L^{2}\right)$-norm and optimal estimate in $l^{2}\left(H^{-1}\right)$-norm. 


\section{Numerical Simulations}

We present some computational experiments, where superconvergence is numerically observed with different numerical schemes. We have considered:

- Domain $\Omega=(0,1) \times(0,1)$ and time interval $(0,1)$, that is $T=1$.

- Exact Solution:

$$
\begin{aligned}
& u_{1}(x, y, t)=e^{-t}(-\cos (2 \pi x) \sin (2 \pi y)+\sin (2 \pi y)), \quad u_{2}(x, y, t)=-u_{1}(y, x, t), \\
& p(x, y, t)=e^{-t} 2 \pi(\sin (2 \pi x)-\sin (2 \pi y))
\end{aligned}
$$

Note that $\nabla \cdot\left(u_{1}, u_{2}\right)=0$ and $\left.\left(u_{1}, u_{2}\right)\right|_{\partial \Omega}=0$. Also $\int_{\Omega} p=0$ and $\left.\nabla p \cdot \mathbf{n}\right|_{\partial \Omega} \neq 0$ where $\mathbf{n}$ is the outward normal vector at $\partial \Omega$

- Time step $k=10^{-4}$. Structurated triangulations of $\Omega$ with $h=\frac{1}{10}, \frac{1}{20}, \frac{1}{30}, \frac{1}{40}$. Since only spatial error must be detected, $k$ must be small enough respect to $h$.

- We compute the discrete error studied in the previous analytical part of this paper, comparing each scheme $\left(u_{h}^{n}, p_{h}^{n}\right)$ with the Stokes projector $S_{h}\left(u\left(t_{n}\right), p\left(t_{n}\right)\right)$.

Three numerical schemes have been considered in the numerical simulations:

1. Euler Semi-Implicit scheme studied in this paper (see subsections 8.1, 8.2 and 8.3):

$$
\left(\delta_{t} u_{h}^{n+1}, v_{h}\right)+a\left(\left(u_{h}^{n+1}, p_{h}^{n+1}\right),\left(v_{h}, q_{h}\right)\right)+c\left(u_{h}^{n}, u_{h}^{n+1}, v_{h}\right)=\left\langle f\left(t_{n+1}\right), v_{h}\right\rangle,
$$

2. Incremental Pressure scheme (see subsection 8.4):

Substep1: Given $\left(u_{h}^{n}, p_{h}^{n}\right) \in V_{h} \times W_{h}$, find $\tilde{u}_{h}^{n+1} \in V_{h}$ solution of

$$
\left\{\begin{array}{l}
\frac{1}{k}\left(\tilde{u}_{h}^{n+1}-u_{h}^{n}, v_{h}\right)+c\left(\tilde{u}_{h}^{n}, \tilde{u}_{h}^{n+1}, v_{h}\right)+\left(\nabla \tilde{u}_{h}^{n+1}, \nabla v_{h}\right)+\left(\nabla p_{h}^{n}, v_{h}\right)=\left\langle f\left(t_{n+1}\right), v_{h}\right\rangle \quad \forall v_{h} \\
\left.\tilde{u}_{h}^{n+1}\right|_{\partial \Omega}=0
\end{array}\right.
$$

Substep2: Given $\left(\tilde{u}_{h}^{n+1}, p_{h}^{n}\right) \in V_{h} \times W_{h}$, find $\left(u_{h}^{n+1}, p_{h}^{n+1}\right) \in V_{h} \times W_{h}$ solution of

$$
\left\{\begin{array}{l}
\frac{1}{k}\left(u_{h}^{n+1}-\tilde{u}_{h}^{n+1}, v_{h}\right)+\left(p_{h}^{n+1}-p_{h}^{n}, v_{h}\right)=0 \quad \forall v_{h} \\
\left(\nabla \cdot u_{h}^{n+1}, p_{h}\right)=0 \quad \forall p_{h} \\
\left.u_{h}^{n+1} \cdot \mathbf{n}\right|_{\partial \Omega}=0
\end{array}\right.
$$

3. Pressure Segregation scheme (see subsection 8.5).

Substep1: Given $\left(p_{h}^{n-1}, u_{h}^{n}\right.$, find $p_{h}^{n} \in W_{h}$ solution of

$$
\left(k \nabla\left(p_{h}^{n}-p_{h}^{n-1}\right), \nabla q_{h}\right)=\left(u_{h}^{n}, \nabla q_{h}\right) \quad \forall q_{h}
$$

Substep2: Given $u_{h}^{n}, p_{h}^{n-1}, p_{h}^{n}$, find $u_{h}^{n+1} \in V_{h}$ solution of

$\frac{1}{k}\left(u_{h}^{n+1}-u_{h}^{n}, v_{h}\right)+c\left(u_{h}^{n}, u_{h}^{n+1}, v_{h}\right)+\left(\nabla u_{h}^{n+1}, \nabla v_{h}\right)+\left(\nabla\left(p_{h}^{n}-p_{h}^{n-1}\right), v_{h}\right)=\left\langle f\left(t_{n+1}\right), v_{h}\right\rangle \quad \forall v_{h}$ 
The absolute errors and convergence rates obtained will be detailed in the following subsections. Here we summarize the results of the simulations:

- $O(h)$-approximation $\left(\mathbb{P}_{1} b / \mathbb{P}_{1}\right.$ or $\left.\mathbb{P}_{2} / \mathbb{P}_{0}\right)$ and semi-implicit Euler scheme.

The numerical results are agreed with the analysis made in this paper, except that we are not able to detect the expected $O(h)$ in $l^{2}\left(H^{1}\right)$-norm for the pressure in the $\mathbb{P}_{1} b / \mathbb{P}_{1}$-approximation and in $l^{2}\left(H^{1}\right)$-norm for the velocity in the $\mathbb{P}_{2} / \mathbb{P}_{0^{-}}$approximation.

- $\mathbb{P}_{2} / \mathbb{P}_{1}$-approximation.

In this case, we detect the orders predicted by the analysis for the velocity and the pressure for the Euler Semi-Implicit scheme. But these orders are also obtained for the splitting schemes. Hence, although for the splitting schemes the analysis is lacking, we conjecture that the same type of results could be deduced.

On the other hand, the $O\left(h^{4}\right)$ (resp. $O\left(h^{2}\right)$ ) order predicted by the analysis is obtained for the velocity (resp. pressure) for the $l^{2}\left(H^{1}\right)$-norm. But these orders are also obtained for the $l^{\infty}\left(L^{2}\right)$-norm, although in this paper only $O\left(h^{3}\right)$ is proved for the velocity and it is not analyzed here for the pressure.

Note that in the Incremental Pressure scheme and the Pressure Segregation scheme, the order for the velocity errors in $L^{2}$-norm, can not be detected for small $h$ due to the influence of the time error.

\subsection{Semi-Implicit Euler with $\mathbb{P}_{1} b / \mathbb{P}_{1}$-approximation}

\begin{tabular}{|c|c|c|c|c|}
\hline \multicolumn{5}{|c|}{ ABSOLUTE ERRORS } \\
\hline $\mathrm{h}$ & 0.0707106 & 0.0353553 & 0.0235702 & 0.0176776 \\
\hline$\left\|S_{h}^{u} u-u_{h}\right\|_{l^{2} L^{2}}$ & 0.02054117586 & 0.00533119776 & 0.002381986923 & 0.001341520128 \\
\hline$\left\|S_{h}^{u} u-u_{h}\right\|_{l^{2} H^{1}}$ & 0.3615287906 & 0.09067109886 & 0.0402638002 & 0.02262494772 \\
\hline$\left\|S_{h}^{u} u-u_{h}\right\|_{l^{\infty} L^{2}}$ & 0.07961372554 & 0.02020235515 & 0.008949038516 & 0.005022097028 \\
\hline$\left\|S_{h}^{p} p-p_{h}\right\|_{l^{2} L^{2}}$ & 0.5332055152 & 0.169360888 & 0.08876683239 & 0.05666878235 \\
\hline$\left\|S_{h}^{p} p-p_{h}\right\|_{l^{\infty} L^{2}}$ & 5.611337315 & 1.373105127 & 0.6056441075 & 0.3402164278 \\
\hline$\left\|S_{h}^{p} p-p_{h}\right\|_{l^{2} H^{1}}$ & 15.31265165 & 10.09500475 & 8.122052658 & 7.006125919 \\
\hline
\end{tabular}

\begin{tabular}{|c|c|c|c|}
\hline \multicolumn{5}{|c|}{ CONVERGENCE RATES } \\
\hline$\left\|S_{h}^{u} u-u_{h}\right\|_{l^{2} L^{2}}$ & 1.9460 & 1.9870 & 1.9957 \\
\hline$\left\|S_{h}^{u} u-u_{h}\right\|_{l^{2} H^{1}}$ & 1.9954 & 2.0021 & 2.0036 \\
\hline$\left\|S_{h}^{u} u-u_{h}\right\|_{l^{\infty} L^{2}}$ & 1.9785 & 2.0082 & 2.0081 \\
\hline$\left\|S_{h}^{p} p-p_{h}\right\|_{l^{2} L^{2}}$ & 1.6546 & 1.5933 & 1.5600 \\
\hline$\left\|S_{h}^{p} p-p_{h}\right\|_{l^{\infty} L^{2}}$ & 2.0309 & 2.0188 & 2.0047 \\
\hline$\left\|S_{h}^{p} p-p_{h}\right\|_{l^{2} H^{1}}$ & 0.6011 & 0.5363 & 0.5138 \\
\hline
\end{tabular}


8.2 Semi-Implicit Euler with $\mathbb{P}_{2} / \mathbb{P}_{0}$-approximation

\begin{tabular}{|c|c|c|c|c|}
\hline \multicolumn{5}{|c|}{ ABSOLUTE ERRORS } \\
\hline $\mathrm{h}$ & 0.0707106 & 0.0353553 & 0.0235702 & 0.0176776 \\
\hline$\left\|S_{h}^{u} u-u_{h}\right\|_{l^{2} L^{2}}$ & 0.160297567 & 0.04282123606 & 0.01938432208 & 0.01099613357 \\
\hline$\left\|S_{h}^{u} u-u_{h}\right\|_{l^{2} H^{1}}$ & 5.709826325 & 2.97360214 & 2.004125509 & 1.510498993 \\
\hline$\left\|S_{h}^{u} u-u_{h}\right\|_{l^{\infty} L^{2}}$ & 0.227696453 & 0.06113359564 & 0.02768846061 & 0.01570631787 \\
\hline$\left\|S_{h}^{p} p-p_{h}\right\|_{l^{2} L^{2}}$ & 0.3563629329 & 0.1542823521 & 0.09760304432 & 0.07061308769 \\
\hline$\left\|S_{h}^{p} p-p_{h}\right\|_{l^{\infty} L^{2}}$ & 4.692005433 & 4.589215029 & 4.306041853 & 3.95470572 \\
\hline
\end{tabular}

\begin{tabular}{|c|c|c|c|}
\hline \multicolumn{4}{|c|}{ CONVERGENCE RATES } \\
\hline$\left\|S_{h}^{u} u-u_{h}\right\|_{l^{2} L^{2}}$ & 1.9044 & 1.9547 & 1.9707 \\
\hline$\left\|S_{h}^{u} u-u_{h}\right\|_{l^{2} H^{1}}$ & 0.9412 & 0.9731 & 0.9829 \\
\hline$\left\|S_{h}^{u} u-u_{h}\right\|_{l^{\infty} L^{2}}$ & 1.8971 & 1.9534 & 1.9708 \\
\hline$\left\|S_{h}^{p} p-p_{h}\right\|_{l^{2} L^{2}}$ & 1.2078 & 1.1293 & 1.1252 \\
\hline$\left\|S_{h}^{p} p-p_{h}\right\|_{l^{\infty} L^{2}}$ & 0.0320 & 0.1571 & 0.2959 \\
\hline
\end{tabular}

8.3 Semi-Implicit Euler with $\mathbb{P}_{2} / \mathbb{P}_{1}$-approximation

\begin{tabular}{|c|c|c|c|c|}
\hline \multicolumn{7}{|c|}{ ABSOLUTE ERRORS } \\
\hline $\mathrm{h}$ & 0.0707106 & 0.0353553 & 0.0235702 & 0.0176776 \\
\hline$\left\|S_{h}^{u} u-u_{h}\right\|_{l^{2} L^{2}}$ & 0.00276963026 & 0.000167065119 & $3.34111872 \mathrm{e}-05$ & $1.23497894 \mathrm{e}-05$ \\
\hline$\left\|S_{h}^{u} u-u_{h}\right\|_{l^{2} H^{1}}$ & 0.180527858 & 0.0238377003 & 0.00718182921 & 0.0030550407 \\
\hline$\left\|S_{h}^{u} u-u_{h}\right\|_{l^{\infty} L^{2}}$ & 0.00423894834 & 0.000261714473 & $5.15274708 \mathrm{e}-05$ & $1.62897169 \mathrm{e}-05$ \\
\hline$\left\|S_{h}^{p} p-p_{h}\right\|_{l^{2} L^{2}}$ & 0.132668315 & 0.0337618908 & 0.0150584711 & 0.00848211006 \\
\hline$\left\|S_{h}^{p} p-p_{h}\right\|_{l^{\infty} L^{2}}$ & 0.199304893 & 0.0511451618 & 0.022854719 & 0.0128857085 \\
\hline$\left\|S_{h}^{p} p-p_{h}\right\|_{l^{2} H^{1}}$ & 0.80348434 & 0.209014013 & 0.0940143439 & 0.053187941 \\
\hline
\end{tabular}

\begin{tabular}{|c|c|c|c|}
\hline \multicolumn{4}{|c|}{ CONVERGENCE RATES } \\
\hline$\left\|S_{h}^{u} u-u_{h}\right\|_{l^{2} L^{2}}$ & 4.0512 & 3.9695 & 3.4596 \\
\hline$\left\|S_{h}^{u} u-u_{h}\right\|_{l^{2} H^{1}}$ & 2.9209 & 2.9589 & 2.9712 \\
\hline$\left\|S_{h}^{u} u-u_{h}\right\|_{l^{\infty} L^{2}}$ & 4.0176 & 4.0081 & 4.0030 \\
\hline$\left\|S_{h}^{p} p-p_{h}\right\|_{l^{2} L^{2}}$ & 1.9744 & 1.9913 & 1.9952 \\
\hline$\left\|S_{h}^{p} p-p_{h}\right\|_{l^{\infty} L^{2}}$ & 1.9623 & 1.9866 & 1.9919 \\
\hline$\left\|S_{h}^{p} p-p_{h}\right\|_{l^{2} H^{1}}$ & 1.9427 & 1.9705 & 1.9800 \\
\hline
\end{tabular}




\subsection{Incremental Pressure with $\mathbb{P}_{2} / \mathbb{P}_{1}$-approximation}

\begin{tabular}{|c|c|c|c|c|}
\hline \multicolumn{5}{|c|}{ ABSOLUTE ERRORS } \\
\hline $\mathrm{h}$ & 0.0707106 & 0.0353553 & 0.0235702 & 0.0176776 \\
\hline$\left\|S_{h}^{u} u-u_{h}\right\|_{l^{2} L^{2}}$ & 0.000344565633 & $2.19641684 \mathrm{e}-05$ & $1.02688172 \mathrm{e}-05$ & $1.24894254 \mathrm{e}-05$ \\
\hline$\left\|S_{h}^{u} u-u_{h}\right\|_{l^{2} H^{1}}$ & 0.021446712 & 0.00280713911 & 0.000849153393 & 0.000387891231 \\
\hline$\left\|S_{h}^{u} u-u_{h}\right\|_{l^{\infty} L^{2}}$ & 0.0013515355 & 0.000100968271 & $3.14616261 \mathrm{e}-05$ & $2.75331122 \mathrm{e}-05$ \\
\hline$\left\|S_{h}^{p} p-p_{h}\right\|_{l^{2} L^{2}}$ & 0.132605783 & 0.033827796 & 0.0151562988 & 0.0086033841 \\
\hline$\left\|S_{h}^{p} p-p_{h}\right\|_{l^{\infty} L^{2}}$ & 0.242004332 & 0.0600466584 & 0.0267550839 & 0.015556087 \\
\hline$\left\|S_{h}^{p} p-p_{h}\right\|_{l^{2} H^{1}}$ & 0.789317037 & 0.208065943 & 0.094618544 & 0.057193705 \\
\hline
\end{tabular}

\begin{tabular}{|c|c|c|c|}
\hline \multicolumn{5}{|c|}{ CONVERGENCE RATES } \\
\hline$\left\|S_{h}^{u} u-u_{h}\right\|_{l^{2} L^{2}}$ & 3.9716 & 1.8751 & -0.6805 \\
\hline$\left\|S_{h}^{u} u-u_{h}\right\|_{l^{2} H^{1}}$ & 2.9336 & 2.9489 & 2.7235 \\
\hline$\left\|S_{h}^{u} u-u_{h}\right\|_{l^{\infty} L^{2}}$ & 3.7426 & 2.8758 & 0.4636 \\
\hline$\left\|S_{h}^{p} p-p_{h}\right\|_{l^{2} L^{2}}$ & 1.9709 & 1.9801 & 1.9684 \\
\hline$\left\|S_{h}^{p} p-p_{h}\right\|_{l^{\infty} L^{2}}$ & 2.0109 & 1.9938 & 1.8850 \\
\hline$\left\|S_{h}^{p} p-p_{h}\right\|_{l^{2} H^{1}}$ & 1.9236 & 1.9435 & 1.7499 \\
\hline
\end{tabular}

\subsection{Pressure segregation with $\mathbb{P}_{2} / \mathbb{P}_{1}$-approximation}

\begin{tabular}{|c|c|c|c|c|}
\hline \multicolumn{5}{|c|}{ ABSOLUTE ERRORS } \\
\hline $\mathrm{h}$ & 0.0707106 & 0.0353553 & 0.0235702 & 0.0176776 \\
\hline$\left\|S_{h}^{u} u-u_{h}\right\|_{l^{2} L^{2}}$ & 0.00034478178 & $2.1004084 \mathrm{e}-05$ & $4.19613214 \mathrm{e}-06$ & $1.53546987 \mathrm{e}-06$ \\
\hline$\left\|S_{h}^{u} u-u_{h}\right\|_{l^{2} H^{1}}$ & 0.021451499 & 0.0028074587 & 0.00084344514 & 0.000358321621 \\
\hline$\left\|S_{h}^{u} u-u_{h}\right\|_{l^{\infty} L^{2}}$ & 0.00142954958 & $9.32110566 \mathrm{e}-05$ & $2.12197877 \mathrm{e}-05$ & $8.75226633 \mathrm{e}-06$ \\
\hline$\left\|S_{h}^{p} p-p_{h}\right\|_{l^{2} L^{2}}$ & 0.132520055 & 0.0337577981 & 0.015064571 & 0.00848449973 \\
\hline$\left\|S_{h}^{p} p-p_{h}\right\|_{l^{\infty} L^{2}}$ & 0.200919704 & 0.0519509245 & 0.0231459756 & 0.0130213259 \\
\hline$\left\|S_{h}^{p} p-p_{h}\right\|_{l^{2} H^{1}}$ & 0.788873864 & 0.207688789 & 0.0937055103 & 0.0531375901 \\
\hline
\end{tabular}

\begin{tabular}{|c|c|c|c|}
\hline \multicolumn{5}{|c|}{ CONVERGENCE RATES } \\
\hline$\left\|S_{h}^{u} u-u_{h}\right\|_{l^{2} L^{2}}$ & 4.0369 & 3.9721 & 3.4946 \\
\hline$\left\|S_{h}^{u} u-u_{h}\right\|_{l^{2} H^{1}}$ & 2.9337 & 2.9658 & 2.9757 \\
\hline$\left\|S_{h}^{u} u-u_{h}\right\|_{l^{\infty} L^{2}}$ & 3.9389 & 3.6500 & 3.0785 \\
\hline$\left\|S_{h}^{p} p-p_{h}\right\|_{l^{2} L^{2}}$ & 1.9729 & 1.9900 & 1.9956 \\
\hline$\left\|S_{h}^{p} p-p_{h}\right\|_{l^{\infty} L^{2}}$ & 1.9514 & 1.9940 & 1.9995 \\
\hline$\left\|S_{h}^{p} p-p_{h}\right\|_{l^{2} H^{1}}$ & 1.9254 & 1.9629 & 1.9719 \\
\hline
\end{tabular}

\section{Conclusions}

Taking into account the interpolation errors, and considering optimal convergence as the same order between the interpolation and discrete errors and superconvergence as extra order in the discrete error with respect to the interpolation one, we can summarize the analytical results of this paper for each approximation as follows: 
- Taylor-Hood approximation $\left(\mathbb{P}_{m} / \mathbb{P}_{m-1}\right.$ with $\left.m \geq 2\right)$ :

- Superconvergence in $l^{2}\left(H^{1}\right)$ and $l^{2}\left(L^{2}\right)$-norms for the velocity, and in $l^{2}\left(H^{1}\right)$-norm for the pressure.

- Optimal convergence in $l^{\infty}\left(L^{2}\right)$ and $l^{\infty}\left(H^{1}\right)$-norms for the velocity.

- $\mathbb{P}_{1}$-iso $\mathbb{P}_{2} / \mathbb{P}_{1}$ and $\mathbb{P}_{1} b / \mathbb{P}_{1}$ approximations:

- Superconvergence in $l^{2}\left(H^{1}\right)$-norm for the velocity.

- Optimal convergence in $l^{\infty}\left(L^{2}\right)$ and $l^{\infty}\left(H^{1}\right)$-norms for the velocity, and in $l^{2}\left(H^{1}\right)$-norm for the pressure.

- $\mathbb{P}_{2} / \mathbb{P}_{0}$ approximation:

Optimal convergence in $l^{2}\left(H^{1}\right)$-norm for the velocity, and in $l^{2}\left(L^{6}\right)$-norm for the pressure.

\section{References}

[1] Brennet, S., Scott, L. The Mathematical Theory of Finite Element Methods, Springer, 2008.

[2] Brezzi, F., Fortin, M. Mixed and Hybrid Finite Element Methods, Springer-Verlag, 1991.

[3] Ciarlet, P.G. The Finite Element Method for Elliptic Problems, North-Holland, 1978.

[4] Ern, A., Guermond, J.L. Theory and Practice of Finite Elements, Springer, 2004.

[5] Fortin, M. Calcul numérique des ecoulements par la méthode des elements finis. Thèse, Université Paris 6, 1972.

[6] Girault, V., Nochetto, N., Scott, R. Estimates of the finite element Stokes projection in $W^{1, \infty}$. C. R. Math. Acad. Sci. Paris 338 (2004), no. 12, 957-962

[7] Girault, V., Raviart, P.-A. Finite element methods for Navier-Stokes equations. Theory and algorithms, Springer-Verlag, 1986.

[8] He, Y., Sun, W. Stability and Convergence of the Crank-Nicolson/Adams-Bashforth scheme for the time-dependet Navier-Stokes equations, SIAM J. Numer. Anal., 452 (2007) 837-869

[9] He, Y. The Euler implicit/explicit scheme for the 2D time-dependent Navier-Stokes equations with smooth or non-smooth initial data., Math. Comput. 77264 (2008) 20972124

[10] Heywood, J.G., Rannacher, R. Finite Element Approximation of the Nonstationary NavierStokes Problem I. Regularity of Solutions and Second-Order Error Estimates for Spatial Discretization, SIAM J. Numer. Anal. 192 (1982) 275-311

[11] Marion, M., Temam, R. Navier-Stokes equations: theory and approximation, Handbook of numerical analysis, Vol. VI, 503-688, North-Holland, 1998.

[12] Prohl, A. Projection and Quasi-Compressibility Methods for Solving the Incompressible Navier-Stokes Equations, B.G. Teubner Stuttgart, 1997. 
[13] Stenberg, R. A technique for analysing finite element methods for viscous incompressible flow. , Internat. J. Numer. Methods Fluids 11 (1990) 935-948

[14] Temam, R. Navier-Stokes equations. Theory and numerical analysis, North-Holland, 1984.

[15] Wang, J., Ye, X. Superconvergence of Finite Elements Aproximations for the Stokes Problem by Projection Methods, SIAM J. Numer. Anal., 39 (2001) 1001-1013

[16] Wheeler, M.F. A priori $L^{2}$ error estimates for Galerkin approximations to parabolic partial differential equations, SIAM J. Numer. Anal., 10 (1973) 723-759 